\title{
Banchoff's sphere and branched covers over the trefoil
}

\author{
Álvaro Lozano Rojo ${ }^{1,2}$ • Rubén Vigara ${ }^{1,2}$
}

Received: 5 July 2017 / Accepted: 16 November 2017

(C) Springer-Verlag Italia S.r.1., part of Springer Nature 2017

\begin{abstract}
A filling Dehn surface in a 3-manifold $M$ is a generically immersed surface in $M$ that induces a cellular decomposition of $M$. Given a tame link $L$ in $M$, there is a filling Dehn sphere of $M$ that "trivializes" (diametrically splits) it. This allows to construct filling Dehn surfaces in the coverings of $M$ branched over $L$. It is shown that one of the simplest filling Dehn spheres of $S^{3}$ (Banchoff's sphere) diametrically splits the trefoil knot. Filling Dehn spheres, and their Johansson diagrams, are constructed for the coverings of $S^{3}$ branched over the trefoil. The construction is explained in detail. Johansson diagrams for generic cyclic coverings and for the simplest locally cyclic and irregular ones are constructed explicitly, providing new proofs of known results about cyclic coverings and the 3 -fold irregular covering over the trefoil.
\end{abstract}

Keywords 3-Manifold · Immersed surface $\cdot$ Filling Dehn surface $\cdot$ Link $\cdot$ Knot $\cdot$ Branched covering

Mathematics Subject Classification Primary 57N10 - 57N35

\section{Introduction}

Filling Dehn surfaces and their Johansson diagrams were introduced in [19], following ideas of [10], as a new way to represent closed orientable 3-manifolds. After [19] some works have appeared on the subject $[1,13,15,16,25-27]$.

To Maite.

$凶$ Rubén Vigara

rvigara@unizar.es

Álvaro Lozano Rojo

alozano@unizar.es

1 Centro Universitario de la Defensa Zaragoza, Academia General Militar, Carretera de Huesca s/n., 50090 Zaragoza, Spain

2 IUMA, Universidad de Zaragoza, Zaragoza, Spain

Published online: 21 November 2017 
In [14], the authors propose a general framework in which filling Dehn surfaces can be applied to knot theory. Any knot (or link) in any 3-manifold can be nicely intersected (split) by a filling Dehn sphere. This filling Dehn sphere appears to be an interesting tool for studying the branched coverings over the knot, because the splitting sphere has "nice lifts" to these branched coverings, in a similar way as the Heegaard surface of a $(g, 1)$-decomposition of the knot $[4,6,7]$. In [14], this is exemplified with the simplest of all knots: the unknot. The techniques of [14] are applied here to the next knot in increasing complexity after the unknot: the trefoil knot.

In Sect. 2 we introduce the basic definitions and notations about filling Dehn surfaces. Sect. 3 recalls the tools introduced in [14]. For more details on this subject see $[14,16]$ and references therein.

In Sect. 4 it is shown that one of the simplest filling Dehn spheres of $S^{3}$ (Banchoff's sphere) splits the trefoil knot. This is used in the subsequent sections to study covers of $S^{3}$ branched over the trefoil. In Sect. 5 we study the cyclic branched covers, obtaining a new proof of Theorem 2.1 of [5] that asserts that the 3-manifolds introduced in [23] coincide with the cyclic branched covers of the trefoil knot. Section 6 deals with other type of coverings, namely locally cyclic (Sect. 6.1) and irregular ones (Sect. 6.2). In particular, in Sect. 6.2 we give another proof of the well known result that asserts that the irregular 3-fold covering of $S^{3}$ branched over the trefoil is $S^{3}[3,9,17]$.

\section{Dehn surfaces and their Johansson's diagrams}

Throughout the paper all 3-manifolds are assumed to be orientable and closed, that is, compact connected and without boundary. On the contrary, surfaces are assumed to be compact, orientable and without boundary, but they could be disconnected. All objects are assumed to be in the smooth category: manifolds have a differentiable structure and all maps are smooth.

Let $M$ be a 3-manifold.

A subset $\Sigma \subset M$ is a Dehn surface in $M$ [20] if there exists a surface $S$ and a general position immersion $f: S \rightarrow M$ such that $\Sigma=f(S)$. If this is the case, the surface $S$ is the domain of $\Sigma$ and it is said that $f$ parametrizes $\Sigma$. If $S$ is a 2-sphere, then $\Sigma$ is a Dehn sphere.

Let $\Sigma$ be a Dehn surface in $M$ and consider a parametrization $f: S \rightarrow M$ of $\Sigma$. The singularities of $\Sigma$ are the points $x \in \Sigma$ such that $\# f^{-1}(x)>1$, and they are divided into double points where two sheets of $\Sigma$ intersect transversely $\left(\# f^{-1}(x)=2\right)$, and triple points where three sheets of $\Sigma$ intersect transversely $\left(\# f^{-1}(x)=3\right)$. The singularities of $\Sigma$ form the singularity set $S(\Sigma)$ of $\Sigma$. We denote by $T(\Sigma)$ the set of triple points of $\Sigma$. The connected components of $S(\Sigma)-T(\Sigma), \Sigma-S(\Sigma)$ and $M-\Sigma$ are the edges, faces and regions of $\Sigma$, respectively.

In the following a curve in $S, \Sigma$ or $M$ is the image of an immersion from $\mathbb{S}^{1}$ or $\mathbb{R}$ into $S$, $\Sigma$ or $M$, respectively. A double curve of $\Sigma$ is a curve in $M$ contained in $S(\Sigma)$.

The preimage under $f$ of the singularity set of $\Sigma$, together with the information about how its points become identified by $f$ in $\Sigma$ is the Johansson diagram $\mathscr{D}$ of $\Sigma$, see [12,19]. Two points of $S$ are related by $f$ if they project onto the same point of $\Sigma$.

Since $S$ is compact and without boundary, double curves are closed and there is a finite number of them, and the number of triple points is also finite. Since $S$ and $M$ are orientable, the preimage under $f$ of a double curve of $\Sigma$ is the union of two different closed curves in $S$. These two curves are sister curves of $\mathscr{D}$. Thus, the Johansson diagram of $\Sigma$ is composed 
of an even number of different closed curves in $S$. We identify $\mathscr{D}$ with the set of different curves that compose it. For any curve $\alpha \in \mathscr{D}$ we denote by $\tau \alpha$ the sister curve of $\alpha$ in $\mathscr{D}$. This defines a free involution $\tau: \mathscr{D} \rightarrow \mathscr{D}$, the sistering of $\mathscr{D}$, that sends each curve of $\mathscr{D}$ to its sister curve in $\mathscr{D}$.

The curves of $\mathscr{D}$ transversely meet others or themselves at the crossings of $\mathscr{D}$. The crossings of $\mathscr{D}$ are the preimage under $f$ of the triple points of $\Sigma$. If $P$ is a triple point of $\Sigma$, the three crossings of $\mathscr{D}$ in $f^{-1}(P)$ form the triplet of $P$.

The Dehn surface $\Sigma \subset M$ fills $M$ if it defines a cell-decomposition of $M$ whose 0-, 1and 2-dimensional skeletons are $T(\Sigma), S(\Sigma)$, and $\Sigma$ respectively [19]. If $\Sigma$ fills $M$ and the domain $S$ of $\Sigma$ is connected, then it is possible to build $M$ out of the Johansson diagram $\mathscr{D}$ of $\Sigma$. Since every 3-manifold has a filling Dehn sphere [19], Johansson diagrams of filling Dehn spheres represent all closed orientable 3-manifolds.

A special case of filling Dehn surfaces is when the domain $S$ of the filling Dehn surface $\Sigma$ is a disjoint union of 2-spheres. In this case, we say that $\Sigma$ is a filling collection of spheres.

\section{Splitting knots with filling Dehn spheres}

In the following paragraphs we summarize some definitions and results from [14]. Results are stated without proof.

Let $L$ be a tame knot or link in a 3-manifold $M$, and let $\Sigma$ be a filling Dehn surface of $M$.

Definition 1 The Dehn surface $\Sigma$ splits $L$ if:

1. $L$ intersects $\Sigma$ transversely in a finite set of non-singular points of $\Sigma$;

2. for each region $R$ of $\Sigma$, if the intersection $R \cap L$ is non-empty it consists exactly of one arc, unknotted in $R$; and

3. for each face $F$ of $\Sigma$, the intersection $F \cap L$ contains at most one point.

The Dehn surface $\Sigma$ diametrically splits $L$ if it splits $L$ and it intersects each connected component of $L$ exactly twice.

Theorem 1 There is a filling Dehn sphere of $M$ that diametrically splits $L$.

In the remainder of this section we assume that $\Sigma$ splits $L$.

Our interest in filling Dehn surfaces that (diametrically) split knots relies on the following result. Let $p: \widehat{M} \rightarrow M$ be a finite sheeted branched covering with downstairs branching set $L$, and take $\widehat{\Sigma}=p^{-1}(\Sigma)$.

Theorem 2 The Dehn surface $\widehat{\Sigma}$ fills $\widehat{M}$. Moreover, if L is a knot and $\Sigma$ is a filling Dehn sphere that diametrically splits $L$ then $\widehat{\Sigma}$ is a filling collection of spheres in $\widehat{M}$, and $\widehat{\Sigma}$ is a Dehn sphere if and only if $p$ is locally cyclic.

Recall that an $n$-fold covering $p$ branched over a knot $L$ is locally cyclic if its monodromy map $\rho$ sends knot meridians onto $n$-cycles [22, p. 209]. This is equivalent to say that $p$ : $p^{-1}(L) \rightarrow L$ is a homeomorphism.

For the study of branched coverings over $L$ it is essential to know its group, i.e. the fundamental group of $M-L$. This can be done also using $\Sigma$. If $R_{1}, \ldots, R_{m}$ are all the different regions of $\Sigma$ disjoint from $L$ and we take a point $Q_{i}$ in each of these regions, $\Sigma-L$ is a strong deformation retract of $M-\left(L \cup\left\{Q_{1}, \ldots, Q_{m}\right\}\right)$. Hence

Proposition 1 The fundamental groups of $M-L$ and $\Sigma-L$ are isomorphic. 
If $f: S \rightarrow M$ is a parametrization of $\Sigma$, the pair $\left(\mathscr{D}, f^{-1}(L)\right)$, where $\mathscr{D}$ is the Johansson diagram of $\Sigma$, is a Johansson diagram of $L$.

Proposition 2 The pair $(M, L)$ can be recovered from a Johansson diagram of $L$. In particular, if $L^{\prime}$ is a link in a 3-manifold $M^{\prime}$ such that $L$ and $L^{\prime}$ have identical Johansson diagrams, there is a homeomorphism between $M$ and $M^{\prime}$ that maps $L$ onto $L^{\prime}$.

Thus, all the information about $L$ is codified in its Johansson diagram.

A presentation of the fundamental group of a Dehn surface in terms of its Johansson diagram was introduced in [15] (cf. [16]). Although the presentation given there is stated for Dehn surfaces of genus $g$, it is not difficult to prove that it is valid in a more general context, including the case of $\Sigma-L$ where the domain surface is a punctured sphere. The generators of this presentation are of two kinds: surfacewise generators and $\mathscr{D}$-dual generators.

Set $M_{L}:=M-L$ and $S_{L}:=S-f^{-1}(L)$. Take a non-singular point $x$ of $\Sigma$ as the base point of the fundamental group $\pi_{L}:=\pi_{1}\left(M_{L}, x\right)$ of $M_{L}$. We also denote by $x$ the preimage of $x$ under $f$, and we choose it as the base point of the fundamental group $\pi_{S_{L}}:=\pi_{1}\left(S_{L}, x\right)$ of $S_{L}$.

The surfacewise generators of $\pi_{L}$ are obtained by pushing forward a generating set of $\pi_{S_{L}}$ to $M$ through $f$ : if $\gamma_{1}, \ldots, \gamma_{k}$ are representatives of a set of generators of $\pi_{S_{L}}$, then $f \circ \gamma_{1}, \ldots, f \circ \gamma_{k}$ are representatives of a set of surfacewise generators of $\pi_{L}$.

Let $\alpha$ and $\beta$ be sister curves of $\mathscr{D}$. Consider two paths $a$ and $b$ in $S_{L}$ starting at $x$ and ending at points on $\alpha$ and $\beta$ respectively. Assume that the endpoints of $a$ and $b$ are related by $f$ and that they are not crossings of $\mathscr{D}$. Then, $\alpha^{\#}=(f \circ a)(f \circ b)^{-1}$ is a loop in $\Sigma-L$ which is said to be dual to $\alpha$. Different choices of $a$ and $b$ provide different dual loops of $\alpha$, but all of them are conjugated by products of surfacewise generators [15]. The inverse loop $\beta^{\#}=\left(\alpha^{\#}\right)^{-1}=(f \circ b)(f \circ a)^{-1}$ is dual to $\beta$.

After repeating this construction for each pair of sister curves of $\mathscr{D}$ we obtain the set $\mathscr{D}^{\#}$ of $\mathscr{D}$-dual generators of $\pi_{L}$.

Proposition 3 [15] Surfacewise generators and $\mathscr{D}$-dual generators generate $\pi_{L}$.

Thus, surfacewise and $\mathscr{D}$-dual generators lead to a presentation of $\pi_{L}$. The relators associated to this presentation are detailed in [15].

If $p: \widehat{M} \rightarrow M$ is an $n$-fold $(n<\infty)$ covering of $M$ branched over $L$, according to Theorem 2, $\Sigma$ lifts to a filling Dehn surface $\widehat{\Sigma}$ of $\widehat{M}$. We want to construct the Johansson diagram $\widehat{\mathscr{D}}$ of $\widehat{\Sigma}$. This construction is specified in Algorithm 3.4 of [14]. The presentation of $\pi_{L}$ in terms of surfacewise and $\mathscr{D}$-dual generators fits quite well to this purpose. In short form:

- surfacewise generators of $\pi_{L}$ allow to construct the domain surface $\widehat{S}$ of $\widehat{\Sigma}$; and

- $\mathscr{D}$-dual generators allow to decide the sistering between the curves of $\widehat{\mathscr{D}}$.

By [15], there is a commutative diagram

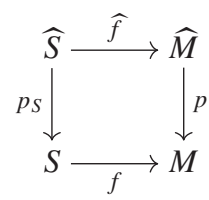

where $\widehat{f}$ is a parametrization of $\widehat{\Sigma}$, and $p_{S}$ is an $n$-fold branched covering with branching set $f^{-1}(L)$. 
Take $p^{-1}(x)=\left\{x_{1}, \ldots, x_{n}\right\}$, and also denote by $\left\{x_{1}, \ldots, x_{n}\right\}$ the corresponding points in $\widehat{S}$. If $\rho: \pi_{L} \rightarrow \Omega_{n}$ is the monodromy homomorphism associated with $p$, where $\Omega_{n}$ is the group of permutations of the set $\left\{x_{1}, \ldots, x_{n}\right\}$, the monodromy homomorphism $\rho_{S}: \pi_{S_{L}} \rightarrow$ $\Omega_{n}$ associated with $p_{S}$ verifies $\rho_{S}=\rho \circ f_{*}$, where $f_{*}: \pi_{S_{L}} \rightarrow \pi_{L}$ is the homomorphism induced by $f$.

Since $f_{*}$ sends a set of generators of $\pi_{S_{L}}$ onto the surfacewise generators of $\pi_{L}, \rho_{S}$ is essentially the same as $\rho$ restricted to the surfacewise generators of $\pi_{L}$. Hence, the knowledge of $\rho$ allows to construct $\widehat{S}$.

Once $\widehat{S}$ is constructed, the curves of $\widehat{\mathscr{D}}$ are the lifts to $\widehat{S}$ of the curves of $\mathscr{D}$. Consider the pair of sister curves $\alpha$ and $\beta$ of $\mathscr{D}$ and their associated paths $a$ and $b$ as before. Let $a_{i}$ and $b_{i}$ be the lifts of $a$ and $b$ respectively to $\widehat{S}$ based at $x_{i}$, and let $\alpha_{i}$ and $\beta_{i}$ be the lifts of $\alpha$ and $\beta$ passing through the endpoint of $a_{i}$ and $b_{i}$ respectively, with $i=1, \ldots, n$. The monodromy map $\rho$ assigns to $\alpha^{\#}$ the permutation $\rho\left(\alpha^{\#}\right)$ of $\left\{x_{1}, \ldots, x_{n}\right\}$ given by

$$
\rho\left(\alpha^{\#}\right)\left(x_{i}\right)=x_{j} \Longleftrightarrow \text { the lift of } \alpha^{\#} \text { starting at } x_{i} \text { ends at } x_{j} .
$$

By the construction of $\alpha^{\#}$, the right-hand side of the previous equivalence is indeed equivalent to say that the endpoints of $a_{i}$ and $b_{j}$ are related by $\widehat{f}$. Therefore, $\alpha_{i}$ and $\beta_{j}$ are sister curves in $\widehat{\mathscr{D}}$ and they must be identified in such a way that the endpoints of $a_{i}$ and $b_{j}$ are related by $\widehat{f}$. Hence, $\rho\left(\alpha^{\#}\right)$ tells us how the lifts of $\alpha$ to $\widehat{S}$ must be identified with the lifts of $\beta$ to $\widehat{S}$. Repeating the same argument for the rest of $\mathscr{D}$-dual generators, all the identifications between the curves of $\widehat{\mathscr{D}}$ are established.

\section{Banchoff's sphere and the trefoil knot}

Let $K$ be the trefoil knot lying in $S^{3}$ with a $2 \pi / 3$ rotational symmetry as in Fig. 1a. A filling Dehn sphere in $S^{3}$ that diametrically splits $K$ can be constructed as follows. Look at the embedded 2-sphere of Fig. 1a, whose interior intersects $K$ in an unknotted arc, and take another two copies of it, each one located at each "petal" of the trefoil knot. These three embedded 2-spheres in $S^{3}$ intersect themselves and $K$ as in Fig. 1b, and they form a filling collection of spheres $\Theta_{0}$ in $S^{3}$. Two Banchoff type 1 surgeries [2] between the 2-spheres transform this filling collection of spheres into a filling Dehn sphere $\Theta$ of $S^{3}$ (see [24]) which is called Banchoff's sphere in [16,27]. According to [16,27], it is one of the three unique filling Dehn spheres of $S^{3}$ with only two triple points. Moreover, if the two surgeries are taken following the knot $K$, as indicated in Fig. 1c, then $\Theta$ diametrically splits $K$. Figure 2 shows how its Johansson diagram $\mathscr{D}$ is obtained from the singular set of $\Theta_{0}$. The dots in the

(a)

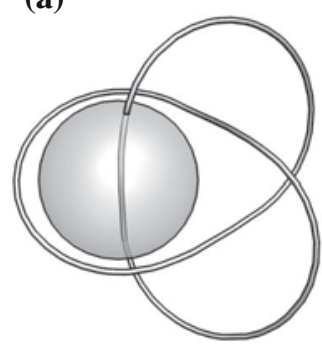

(b)

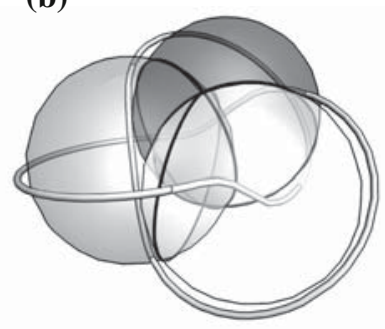

(c)

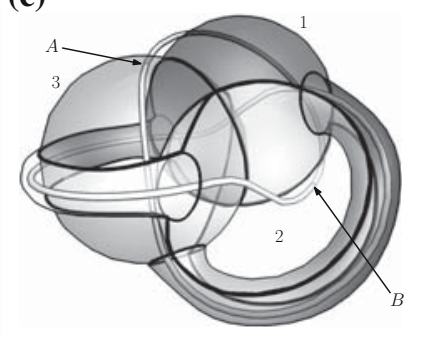

Fig. 1 Banchoff's sphere splitting the trefoil 
(a)

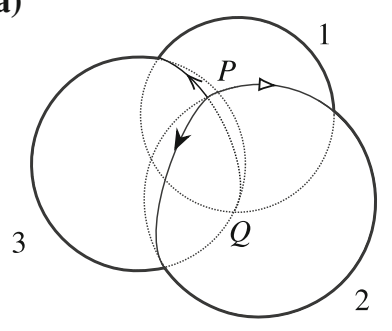

(b)

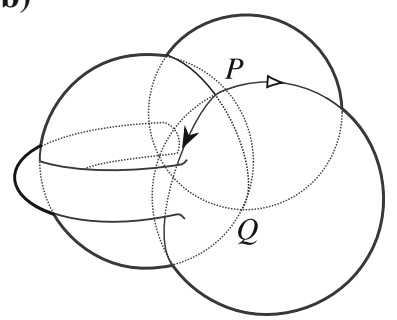

(c)

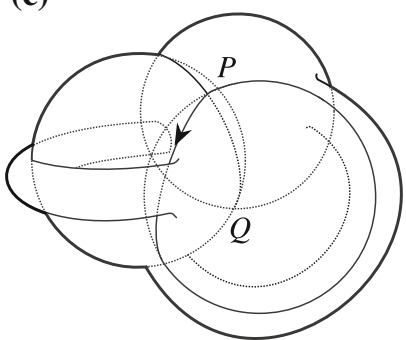

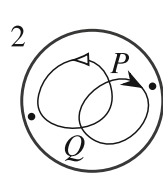
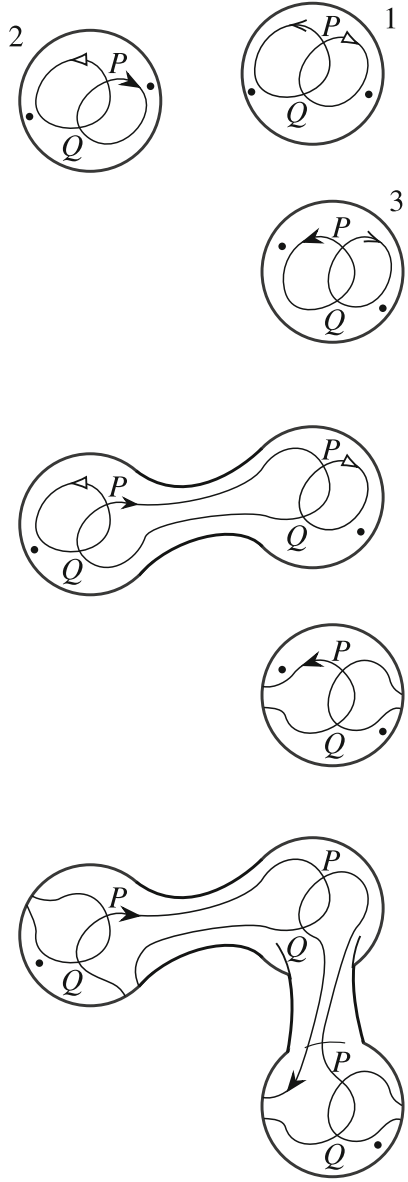

Fig. 2 Constructing Banchoff's sphere: a starting from a bunch of three 2-spheres, b, $\mathbf{c}$ we add tubes to build a Dehn sphere. In the right column we can see how the corresponding diagram changes

right-hand side of the picture represent the intersection of the surface with the trefoil knot. Figure 3 a shows the usual representation of $\mathscr{D}$ where one point of the sphere has been sent to infinity.

Let $f: S^{2} \rightarrow S^{3}$ be a parametrization of $\Theta$. The two curves $\alpha, \beta$ of the diagram $\mathscr{D}$ verify $\beta=\tau \alpha$, and they must be identified following the arrows in the obvious way. The preimages by $f$ of the two intersection points $A, B$ of $\Theta$ with $K$ are the points also denoted by $A, B$ in $S^{2}$, see Fig. 3a. When the notation does not lead to confusion, we will use the same names for the objects in $\Theta$ and their preimages in $S^{2}$.

The fundamental group $\pi_{1}(\Theta-\{A, B\}, x) \simeq \pi_{K}$ based at the point $x$ is generated by the loops $m$ and $c$, where:

- $m$ is the generator of $\pi_{1}\left(S^{2}-\{A, B\}\right)$ depicted in Fig. 3a; and

- the loop $c=a b^{-1}=\alpha^{\#}$ dual to the curve $\alpha$ of $\mathscr{D}$, where $a$ and $b$ are the paths depicted in Fig. 3a joining $x$ with two related points on $\alpha$ and $\beta$ respectively.

Note that the loop $m$ in $\Theta$ is homotopic to a meridian of $K$. 
(a)

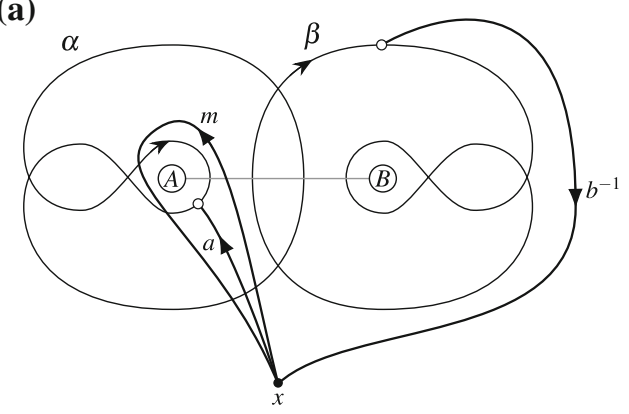

(b)

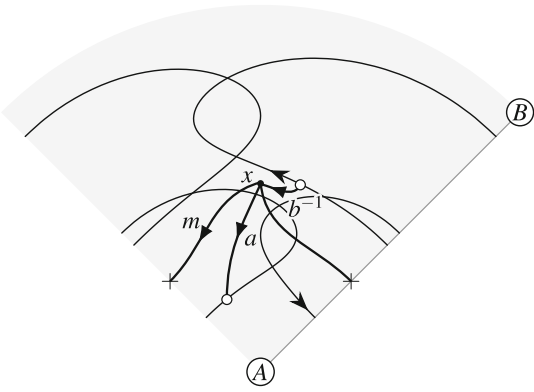

Fig. 3 a Banchoff's sphere diagram with the loops selected to generate the fundamental group of the trefoil knot complement. b The fan $\Delta$ obtained by cutting the previous diagram along the thin horizontal line between point $A$ and $B$

After computing for $\Theta-\{A, B\}$ the presentation of its fundamental group given in [15], we obtain

$$
\pi_{K} \simeq\left\langle m, c \mid m c m=\mathrm{cm}^{-1} c\right\rangle .
$$

It is straightforward to see that this group is isomorphic to the trefoil knot group.

\section{Cyclic branched covers over the trefoil knot}

\subsection{Johansson diagrams and fundamental group}

Let $p: \widehat{M}_{n} \rightarrow S^{3}$ be the $n$-fold $(n<\infty)$ cyclic covering of $S^{3}$ branched over $K$. By Theorem 2, Banchoff's sphere $\Theta \subset S^{3}$ lifts to a filling Dehn sphere $\widehat{\Theta}$ of $\widehat{M}_{n}$.

We use the same notation as in Sect. 3. Take $p^{-1}(x)=\left\{x_{1}, \ldots, x_{n}\right\}$, and denote also by $\left\{x_{1}, \ldots, x_{n}\right\}$ the corresponding points in $\widehat{S}$. Let $\rho: \pi_{K} \rightarrow \Omega_{n}$ be the monodromy homomorphism associated with $p$. Since $p$ is cyclic, $\rho$ sends $\pi_{K}$ onto a cyclic subgroup $C_{n}$ of $\Omega_{n}$. The fact that $C_{n}$ is abelian and the relation in (1) imply that $\rho(c)=\rho(m)^{3}$. Therefore $C_{n}=\langle\rho(m)\rangle$. Since $C_{n}$ must act transitively on $\left\{x_{1}, \ldots, x_{n}\right\}, \rho(m)$ must be a cycle of order $n$. If we identify $\Omega_{n}$ with the permutation group of the subscripts $\{1, \ldots, n\}$ in the natural way, renaming $\left\{x_{1}, \ldots, x_{n}\right\}$ if necessary, we can assume that $\rho(m)=(1,2, \ldots, n)$. In the following paragraphs all the subscripts are considered modulo $n$.

The loop $m$ generates the fundamental group of $S-\{A, B\}$, which is infinite cyclic. Therefore, the monodromy homomorphism $\rho_{S}$ is given by $m \mapsto(1,2, \ldots, n)$. If $m_{i}$ is the lift of $m$ to $\widehat{S}$ based at $x_{i}$, by the choice of $\rho(m)$, the lifted path $m_{i}$ starting at $x_{i}$ must have its endpoint at $x_{i+1}$.

After: (i) cutting the diagram of Fig. 3 a along the line that connects $A$ and $B$ in the same figure; and (ii) sending $B$ to infinity; the fan $\Delta$ of Fig. 3 b is obtained. The domain $\widehat{S}$ of $\widehat{\Theta}$ is obtained by cyclically gluing $n$ copies of $\Delta$. If $\Delta_{i}$ is the copy of $\Delta$ that contains $x_{i}, i=$ $1, \ldots, n$, the diagram $\widehat{\mathscr{D}}$ of $\widehat{\Theta}$ is built up with $\Delta_{1}, \ldots, \Delta_{n}$ glued together counterclockwise (the direction does not matter, but we choose it according to the direction of $m$ in the diagram to visualize it better, see Fig. 4).

As explained in Sect. 3, the lifts of $c$ to $\widehat{\Theta}$ describe how the curves of the diagram $\widehat{\mathscr{D}}$ become identified in $\widehat{\Theta}$. Let $a_{i}, b_{i}$ and $c_{i}$ be the lifts of $a, b$ and $c$ respectively based at $x_{i}$. Let $\alpha_{i}$ be the lift of $\alpha$ to $\widehat{S}$ where $a_{i}$ has its endpoint. In the same way, let $\beta_{i}$ be the lift of $\beta$ to 


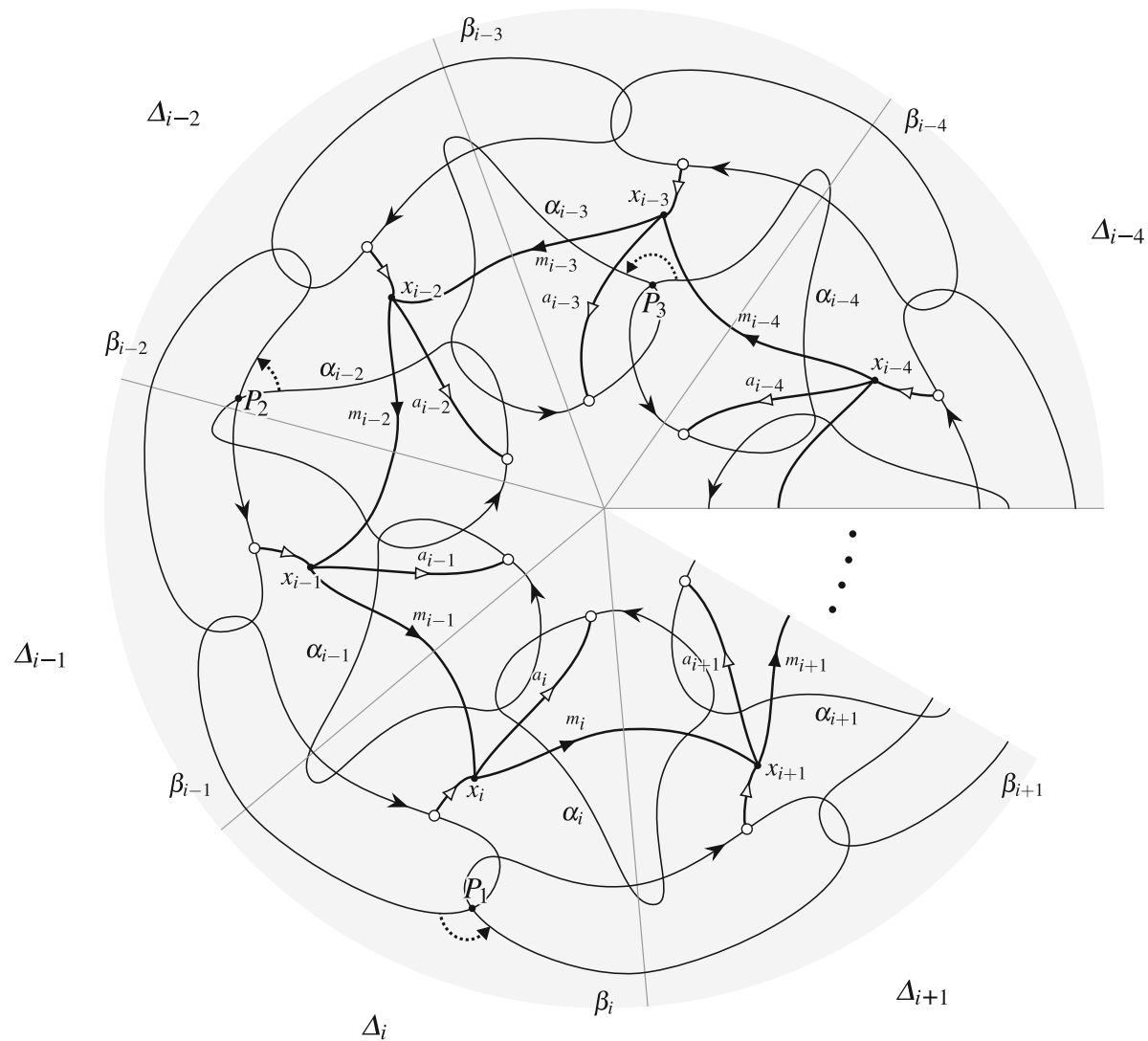

Fig. 4 Building the diagram of a cyclic branched covering. The lifts of $m$ are the thick paths marked with triangle arrows. Those of $a$ are the thick paths marked with triangle empty arrows. The lifts of $b$ are unlabelled, but the path $b_{i}^{-1}$ is the one ending at $x_{i}$ also marked with an empty triangle arrow

$\widehat{S}$ where $b_{i}$ has its endpoint. With this notation, the situation in $\widehat{S}$ is as depicted in Fig. 4. The path $c_{i}$ connects $x_{i}$ with $x_{\rho(c)(i)}=x_{i+3}$, crossing a double curve of $\widehat{\Theta}$. Hence, the curve at which $a_{i}$ ends must be identified with the one at which $b_{i+3}^{-1}$ starts and therefore $\tau \alpha_{i}=\beta_{i+2}$ (see Fig. 4). This allows us to proof:

Lemma 1 The fundamental group of $\widehat{M}_{n}$ is isomorphic to the Sieradski group

$$
\left.\mathscr{S}(n)=\left\langle g_{1}, \ldots, g_{n}\right| g_{i}=g_{i-1} g_{i+1} \text { for } i=1, \ldots, n\right\rangle,
$$

where the indices are taken modulo $n$.

Proof The fundamental group of $\widehat{M}_{n}$ coincides with that of $\widehat{\Theta}$. Since $\widehat{S}$ is a 2-sphere, we can use the presentation given in [16, Chp. 4]. In this presentation the fundamental group is generated by the dual loops of $\alpha_{i}$ and $\beta_{i}, i=1, \ldots, n$, and the relators are given by the triplets of $\widehat{\mathscr{D}}$. By construction, dual loops of sister curves are inverse to each other, and so $\pi_{1}\left(\widehat{M}_{n}\right)$ is generated just by the dual loops $\beta_{1}^{\#}, \ldots, \beta_{n}^{\#}$. 
(a)

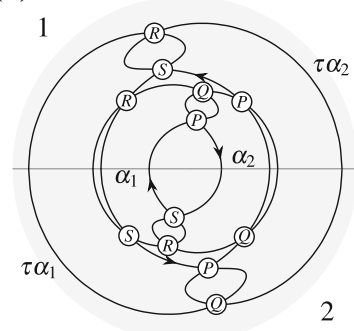

(b)

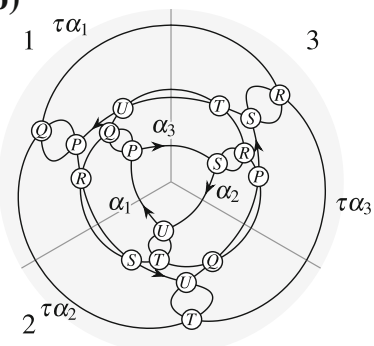

(c)

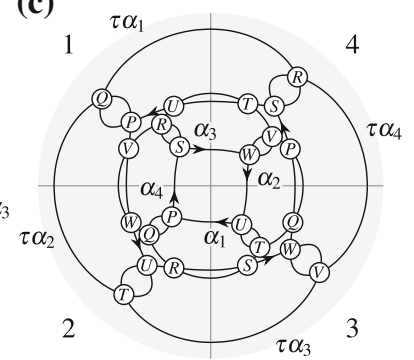

Fig. 5 Diagrams for the first cyclic coverings branched over the trefoil: a the lens space $L(3,1)$ as the cyclic 2 -fold covering of $S^{3}$ branched over $K$; b the 3 -fold covering; and $\mathbf{c}$ the 4 -fold covering

Let us determine the triplets of $\widehat{\mathscr{D}}$. Take the point $P_{1}$ in the $i$-th fan $\Delta_{i}$ of Fig. 4 . The curves $\beta_{i-1}$ and $\beta_{i}$ intersect at $P_{1}$. Since $P_{1}$ is the third crossing after the arrow in $\beta_{i}$, it must be identified with the third crossing after the arrow in $\tau \beta_{i}=\alpha_{i-2}$, which is the point $P_{2}$ in $\Delta_{i-2}$. In the same way, since $P_{2}$ is the fifth crossing after the arrow in $\beta_{i-2}$, it must be identified with the fifth crossing after the arrow in $\tau \beta_{i-2}=\alpha_{i-4}$, which is the point $P_{3}$ in $\Delta_{i-3}$. Finally, since $P_{3}$ is the second crossing after the arrow in $\alpha_{i-3}$, it must be identified with the second crossing after the arrow in $\tau \alpha_{i-3}=\beta_{i-1}$, which is, as expected, $P_{1}$.

For each $j=1,2,3$ take a small path $\delta_{j}$ near $P_{j}$ such that the endpoint of $\delta_{j}$ is related by $\widehat{f}$ with the starting point of $\delta_{j+1}$, where the subscripts are taken modulo 3 (as the dotted arcs in Fig. 4). Then the loop $\left(\widehat{f} \circ \delta_{1}\right)\left(\widehat{f} \circ \delta_{2}\right)\left(\widehat{f} \circ \delta_{3}\right)$ is contractible in $\widehat{\Theta}$. Hence, the product of $\widehat{\mathscr{D}}$-dual loops $\beta_{i}^{\#} \beta_{i-2}^{\#} \alpha_{i-3}^{\#}=\beta_{i}^{\#} \beta_{i-2}^{\#}\left(\beta_{i-1}^{\#}\right)^{-1}$ is also contractible (full details in [16, Chp. 4]). Therefore $\beta_{i-1}^{\#}=\beta_{i}^{\#} \beta_{i-2}^{\#}$ in $\pi_{1}\left(\widehat{M}_{n}, x\right)$.

It is straightforward to see that all the relations are of this form. In [16, Chp. 4] it is proved that these are all the nontrivial relations. Taking $g_{i}=\beta_{n-i}^{\#}, i=1, \ldots, n$, the presentation of $\mathscr{S}(n)$ of the statement is obtained.

Remark 1 Lemma 1 is included in [5] as part of the proof of Theorem 2.1 (Theorem 3 below).

Example 1 Consider $p: \widehat{M}_{2} \rightarrow S^{3}$ given by the presentation $m \mapsto(1,2), c \mapsto(1,2)$, see Fig. 5a. The previous description allows us to conclude that $\pi_{1}\left(\widehat{M}_{2}\right)=\mathbb{Z}_{3}$, in fact $\widehat{M}_{2}$ is $L(3,1)$. Figure 5 b shows the diagram constructed for $\widehat{M}_{3}$ given by the presentation $m \mapsto$ $(1,2,3), c \mapsto 1_{\Omega_{3}}$. The fundamental group is isomorphic to the group of the quaternions $Q_{8}$. Hence, $\widehat{M}_{3}$ is one of the prism manifolds, which are characterized by their fundamental group. According to the notation of [11] this is $M(2,1)$, also called the Quaternionic Space [18]. Consider now the presentation $m \mapsto(1,2,3,4), c \mapsto(1,4,3,2)$, the fundamental group of $\widehat{M}_{4}$ is $S L_{2}\left(\mathbb{Z}_{3}\right) \cong \mathbb{Z}_{3} \rtimes Q_{8}$. This is one of the tetrahedral manifolds, which are also characterized by their fundamental group. In this case the manifold is the Octahedral space [18].

\subsection{The Sieradski complex}

The family of polyhedra with identified faces depicted in Fig. 6 was introduced in [23]. The quotient spaces of these polyhedra is a family of 3-manifolds $M_{n}$, with $n \geq 2$, whose fundamental groups are the Sieradski groups $\mathscr{S}(n)$. In [5] it is proved that $M_{n}$ is the $n$-fold cyclic branched cover over the trefoil knot, $\widehat{M}_{n}$ in our notation. As shown above, Sieradski 
(a)

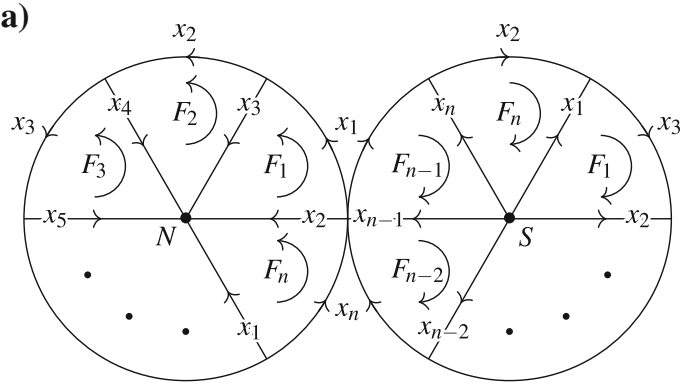

Fig. 6 The Sieradski complex on the sphere $S^{2}=\partial D^{3}$

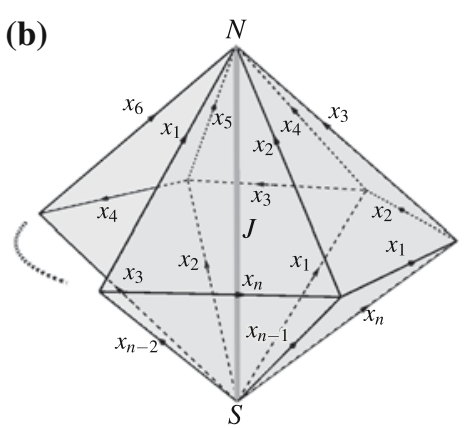

(b)

(c)

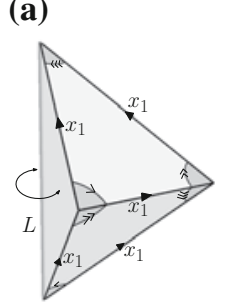

(b)
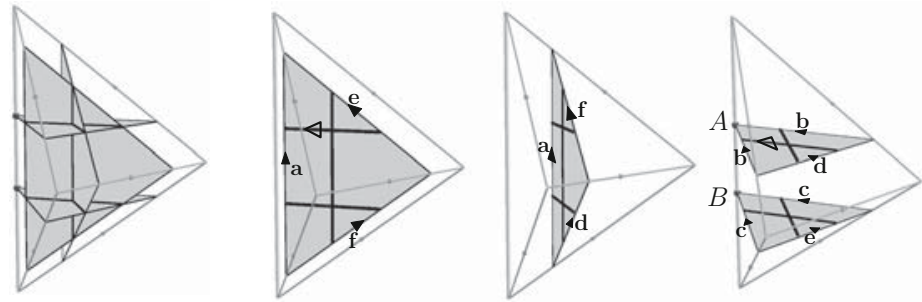

(d)
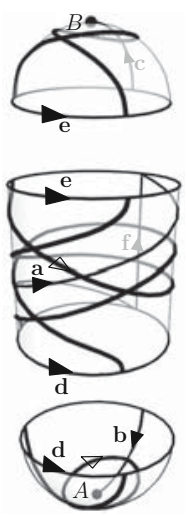

(e)

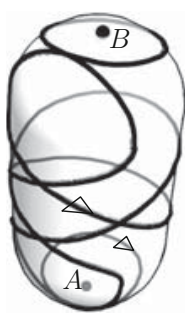

Fig. 7 Banchoff's sphere splitting the trefoil in Sieradski complex for $n=1$

groups naturally appear in our construction, so it is natural to expect that the lifts of Banchoff's sphere have some relation with Sieradski polyhedra (see Fig. 6). In fact, Banchoff's sphere allows us to give an alternative proof of Theorem 2.1 of [5].

Fix an integer $n \geq 2$. For the Sieradski polyhedron $\mathscr{P}_{n}$ of Fig. $6 \mathrm{~b}$, consider the $2 \pi / n$ rotation $r$ around the vertical central axis $J$ connecting the "north and south poles" $N$ and $S$ of $\mathscr{P}_{n}$. By the symmetry of the identification of points on $\partial \mathscr{P}_{n}, r$ preserves these identifications ( $r$ sends identified points to identified points on $\partial \mathscr{P}_{n}$ ), and therefore $r$ defines a homeomorphism of $M_{n}$. Moreover, the group generated by $r$ is cyclic of order $n$. The quotient space of the pair $\left(M_{n}, J\right)$ under the action of $\langle r\rangle$ is the pair $\left(M_{1}, L\right)$, where $M_{1}$ is the manifold 
obtained after identifying the faces of the tetrahedron $\mathscr{P}_{1}$ of Fig. 7 a in the following way: the two vertical faces must be identified by a rotation around the vertical edge; and the other two faces must be identified in the unique way in which the boundary edges become identified as indicated by the arrows. Let $L$ denote the image of the vertical edge of the tetrahedron after the identification.

Lemma 2 The pair $\left(M_{1}, L\right)$ is homeomorphic to $\left(S^{3}, K\right)$.

Proof Take the immersed surface $\Sigma$ in $M_{1}$ which is the projection of the surface depicted inside the tetrahedron in Fig. $7 b$, c. The four pieces of this surface become glued by the identification as it is indicated in Fig. 7d, e, and the intersection of $L$ with $\Sigma$ corresponds to the points $A$ and $B$ of the same figure. After some ambient isotopies, the diagram of $(\Sigma, L)$ becomes the one of Fig. 3a. It is not difficult to check that $\Sigma$ fills $M_{1}$. Therefore, $M_{1}$ is $S^{3}$ and $\Sigma$ is Banchoff's sphere. Since the intersection of $L$ and $\Sigma$ coincides with the intersection of the trefoil and $\Theta$, by Proposition 2 we conclude also that $L$ is the trefoil knot.

Theorem 3 [5, Thm. 2.1] For each $n=2,3, \ldots$ the 3-manifold $M_{n}$ is the $n$-fold cyclic cover of $S^{3}$ branched over the trefoil.

Proof The unique points of $M_{n}$ that become fixed by $r$ are those on $J$. Therefore, the covering of $M_{n}$ over $M_{1}$ defined by $\langle r\rangle$ is a $n$-fold covering of $S^{3}$ branched over $K$. Since the group $\langle r\rangle$ of deck transformations is cyclic of order $n$, it turns out that $M_{n}$ is the $n$-fold cyclic covering of $S^{3}$ branched over the trefoil knot.

The lift of Banchoff's sphere to $M_{n}$ can be built inside $\mathscr{P}_{n}$ by cyclically gluing $n$ copies of the pieces of the surface of Fig. $7 \mathrm{~b}$ around the axis $J$ (Fig. 6b).

\section{Other examples}

\subsection{Locally cyclic branched covers}

Since all locally cyclic coverings of 2 and 3 sheets of $S^{3}$ branched over $K$ are in fact cyclic, the first non-cyclic example $p: M \rightarrow S^{3}$ is the one given by the representation of $\pi_{K}$ into $\Omega_{4}$ defined by $m \mapsto(1,2,3,4)$ and $c \mapsto(1,2)$. The construction of the diagram is as in the cyclic case. Since the image of $m$ is a cycle of maximal length the lift of $\Theta$ has $S^{2}$ as domain, and $\rho(c)$ describes how to identify the curves of the diagram. The final diagram is the one of Fig. 8, which gives

$$
\pi_{1}(M) \cong\left\langle\alpha_{1}, \alpha_{2}, \alpha_{3}, \alpha_{4} \mid \alpha_{1} \alpha_{2}^{-2}, \alpha_{1} \alpha_{3} \alpha_{4}^{-1}, \alpha_{1} \alpha_{3}^{-1} \alpha_{4}, \alpha_{4} \alpha_{2}^{-1} \alpha_{3}\right\rangle \cong \mathbb{Z}_{3} \rtimes \mathbb{Z}_{8} .
$$

Therefore $M$ is the prism manifold $M(3,2)$ in the notation of [11]. Compare it with the 4-fold cyclic covering depicted in Fig. 5c.

\subsection{The 3-fold irregular cover}

Let $p: \widehat{M} \rightarrow S^{3}$ be an $n$-fold branched covering over the trefoil $K$ with $n<\infty$. In the previous sections we have seen how to construct the Johansson diagram of a filling Dehn sphere of $\widehat{M}$ when $\rho(m)$ is a cycle of order $n$, where $\rho$ is the monodromy of $p$. It is also possible to construct the Johansson diagram of a filling Dehn sphere of $\widehat{M}$ in the general case, even if $\rho(m)$ is not a cycle of maximal length. We illustrate this case with the simplest 
Fig. 8 The diagram for the locally cyclic covering given by the presentation $m \mapsto(1,2,3,4), c \mapsto(1,2)$

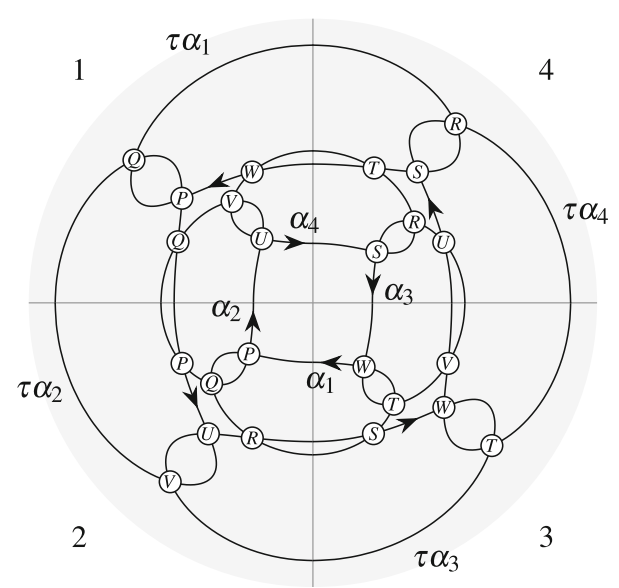

example: the 3 -fold irregular covering over the trefoil. We use the same notation as in previous sections.

Assume that $n=3$ and that $\rho(m)=(12)$. The lifts $m_{1}, m_{2}$ and $m_{3}$ of $m$ to the domain surface $\widehat{S}$ of $\widehat{\Sigma}=p^{-1}(\Theta)$ verify

- $m_{1}$ connects $x_{1}$ with $x_{2}$

- $m_{2}$ connects $x_{2}$ with $x_{1}$; and

$-m_{3}$ connects $x_{3}$ with itself.

Therefore, $\widehat{S}$ is a disjoint union of two 2 -spheres. One of them, $S_{12}$, contains $x_{1}$ and $x_{2}$ and can be obtained by gluing two copies $\Delta_{1}$ and $\Delta_{2}$ of the fan $\Delta$. The other one, $S_{3}$, containing $x_{3}$ must be a copy of the domain surface of $\Theta$. The restriction of $p_{S}$ to $S_{12}$ is a 2-fold branched covering with branching set $\{A, B\}$, and $\left.p_{S}\right|_{S_{3}}$ is a 1-fold branched covering with branching set $\{A, B\}$, hence a homeomorphism. The diagram $\widehat{\mathscr{D}}$ of $\widehat{\Sigma}$ is the lift to $\widehat{S}=S_{12} \sqcup S_{3}$ of the diagram $\mathscr{D}$ through $p_{S}$. By the same arguments of previous sections, $\widehat{\mathscr{D}}$ in $S_{12}$ looks like the left-hand side of Fig. 9a. The diagram $\widehat{\mathscr{D}}$ in $S_{3}$ looks exactly like the diagram $\mathscr{D}$ in $S$, except for the identification of the curves, that will be determined by the element $\rho(c)$ given by the monodromy homomorphism. Set $\Sigma_{12}=\widehat{f}\left(S_{12}\right)$ and $\Sigma_{3}=\widehat{f}\left(S_{3}\right)$.

Since $\rho(m)$ and $\rho(c)$ verify the identity

$$
\rho(m) \rho(c) \rho(m)=\rho(c) \rho(m)^{-1} \rho(c)
$$

and the subgroup of $\Omega_{3}$ generated by $\rho(m)$ and $\rho(c)$ acts transitively on the set $\{1,2,3\}$, then $\rho(c)=(1,3)$ or $\rho(c)=(2,3)$. Assume that $\rho(c)=(2,3)$ (the case $\rho(c)=(1,3)$ is equivalent). The endpoint of $a_{1}$ is related by $\widehat{f}$ with the endpoint of $b_{1}$, the endpoint of $a_{2}$ is related by $\widehat{f}$ with with the endpoint of $b_{3}$, and the endpoint of $a_{2}$ is related by $\widehat{f}$ with the endpoint of $b_{3}$. The resulting sistering of $\widehat{\mathscr{D}}$ is indicated in Fig. 9a (in this figure we depict $S_{3}$ as the fan $\Delta_{3}$ glued with itself, compare it with Fig. 3).

Now, we modify $\widehat{\Sigma}$ by a surgery operation near a triple point where a sheet of $\Sigma_{12}$ and a sheet of $\Sigma_{3}$ intersect. We perform a Banchoff type 1 surgery between $\Sigma_{12}$ and $\Sigma_{3}$ around the triple point $P$ as indicated in the top part of Fig. 10. The local effect of this operation on the diagram is depicted in the bottom part of the same picture. Figure 9a shows how the piping between $\Sigma_{12}$ and $\Sigma_{3}$ is performed: we remove the shaded disks and attach a tube along the dashed arc. The resulting Dehn surface $\widetilde{\Sigma}$ has as domain the connected sum of $S_{12}$ and $S_{3}$, hence it is a Dehn sphere. Its Johansson diagram appears in Fig. $9 \mathrm{~b}$. 

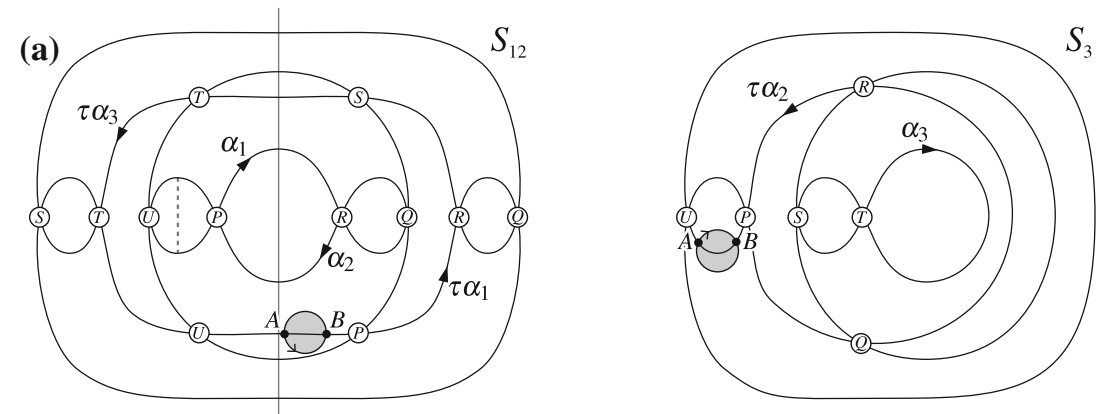

(b)

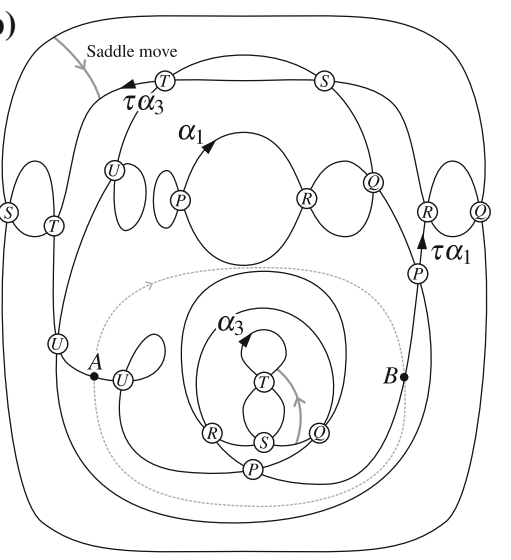

(c)

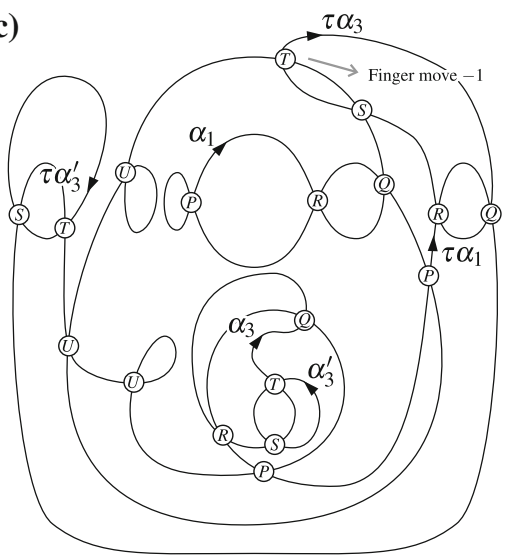

(d)

(e)
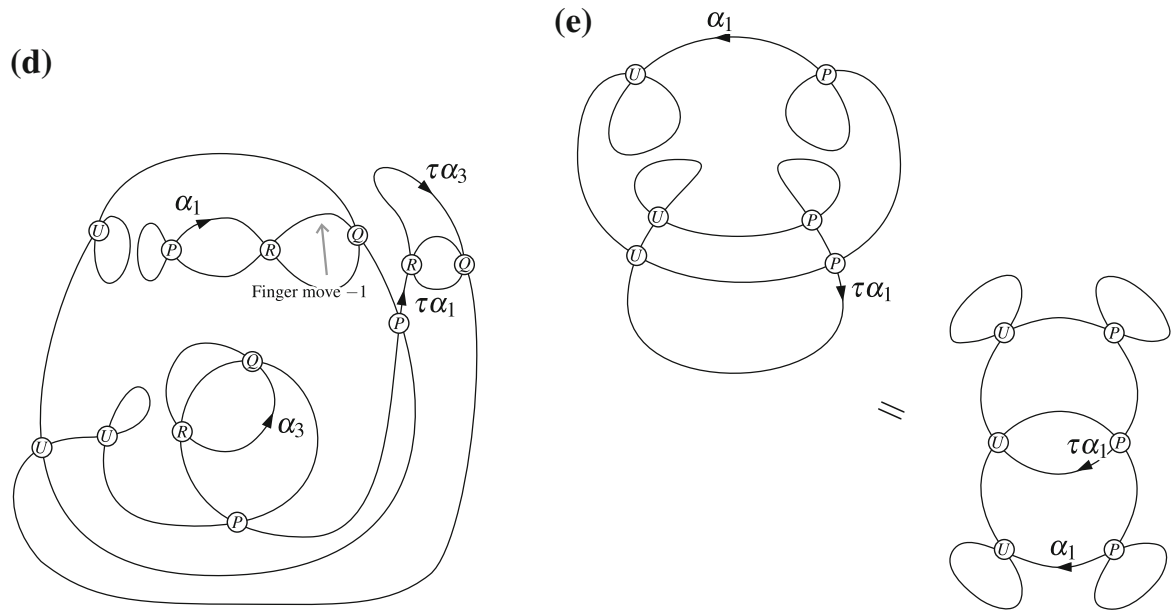

Fig. 9 The first non-locally cyclic cover of $S^{3}$ branched over $K$ is $S^{3}$

Proposition 4 The Dehn sphere $\widetilde{\Sigma}$ fills $\widehat{M}$.

Proof In order to check that $\widetilde{\Sigma}$ fills $\widehat{M}$ it must be proved that all its edges, faces and regions are open 1-, 2- and 3-dimensional disks, respectively. The diagram of $\widetilde{\Sigma}$ implies that the edges and faces of $\widetilde{\Sigma}$ verify this requirement. 
(a)
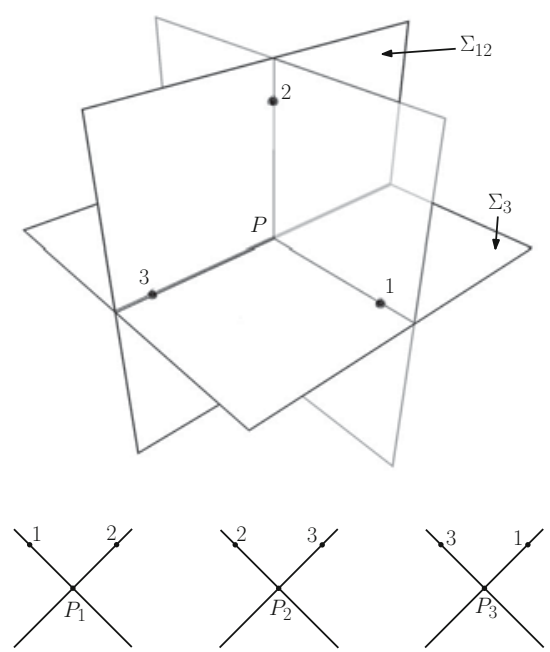

(b)
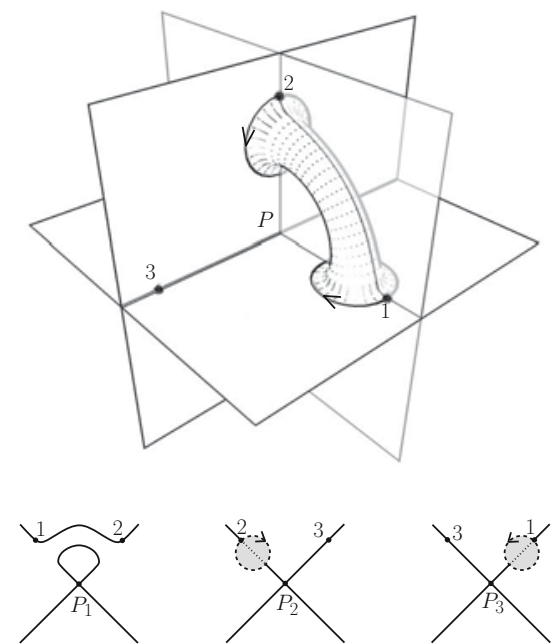

Fig. 10 Surgery around a triple point

The embedded 2-sphere $\widehat{\Sigma}_{3}$ is nullhomotopic because $\Theta$ is nullhomotopic, and therefore $\widehat{\Sigma}_{3}$ is separating in $\widehat{M}$. This implies that the surgery that transforms $\widehat{\Sigma}$ into $\widetilde{\Sigma}$ connects two regions $R_{1}$ and $R_{2}$ of $\widehat{\Sigma}$ on one connected component of $\widehat{M}-\widehat{\Sigma}_{3}$ with other two regions $R_{3}$ and $R_{4}$ of $\widehat{\Sigma}$ on the other connected component of $\widehat{M}-\widehat{\Sigma}_{3}$, creating two regions of $\widetilde{\Sigma}$. If $R_{1}=R_{2}$, there would be a loop $\lambda$ in $\widehat{M}$ that intersects $\widehat{\Sigma}$ transversely only at one non-singular point of $\widehat{\Sigma}$, and in this case $p \circ \lambda$ would intersect $\Theta$ transversely only at one non-singular point of $\Theta$, but this cannot happen because $\Theta$ is nullhomotopic (as any Dehn sphere in $S^{3}$ ). Hence, $R_{1} \neq R_{2}$, and the same argument gives $R_{3} \neq R_{4}$. Therefore, the four regions of $\widehat{\Sigma}$ that become connected in pairs by the surgery are all different, and this implies that all the regions of $\widetilde{\Sigma}$ are open 3-balls.

The Johansson diagrams of two filling Dehn spheres of the same 3-manifold are related by a sequence of $\boldsymbol{f}$-moves [16,26,27], provided that both filling Dehn spheres are nullhomotopic (an equivalent set of moves is proposed in [1]). These $f$-moves give another proof of the following well-known result about the 3-manifold $\widehat{M}$.

Theorem $4[3,8,9,17]^{1}$ The 3-fold irregular branched covering of the trefoil is the 3-sphere.

Proof Starting from the diagram $\widetilde{\mathscr{D}}$ of Fig 9b, after a saddle move the diagram $\mathscr{D}_{1}$ of Fig. 9c is obtained. After a finger move -1 (and ambient isotopies) we get the diagram $\mathscr{D}_{2}$ of Fig $9 \mathrm{~d}$, and another finger move -1 finally gives the diagram $\mathscr{D}_{3}$ of Fig. 9e, which coincides with the diagram of Johansson's sphere, a well-known filling Dehn sphere of $S^{3}$ (see $[14,16]$ ). Since $\mathscr{D}_{3}$ is filling, $\mathscr{D}_{2}$ is also filling because it is obtained by applying a finger move +1 to $\mathscr{D}_{3}$ (see [16, Lem. 5.6 and Thm. 5.20]). The same argument applies to conclude that $\mathscr{D}_{1}$ is also filling. Since $\widetilde{\mathscr{D}}$ and $\mathscr{D}_{1}$ are filling diagrams, the saddle move that relate them is an $f$-move. By [16, Thm. 5.20], $\widehat{M}$ is $S^{3}$.

${ }^{1}$ Of course, these and other similar questions became automatically solved after Perelman's proof of the Poincaré Conjecture [21]. 
Acknowledgements Partially supported by the European Social Fund and Diputación General de Aragón (Grant E15 Geometría) and by MINECO grants MTM2013-46337-C2, MTM2016-77642-C2, MTM201345710-C2 and MTM2016-76868-C2-2-P.

\section{References}

1. Amendola, G.: A local calculus for nullhomotopic filling Dehn spheres. Algebraic Geom. Topol. 9(2), 903-933 (2009)

2. Banchoff, T.F.: Triple points and surgery of immersed surfaces. Proc. Am. Math. Soc. 46(3), 407-413 (1974)

3. Burde, G.: On branched coverings of $S^{3}$. Can. J. Math. 23(1), 84-89 (1971)

4. Cattabriga, A., Mulazzani, M.: Strongly-cyclic branched coverings of $(1,1)$-knots and cyclic presentations of groups. Math. Proc. Camb. Philos. Soc. 135(1), 137-146 (2003)

5. Cavicchioli, A., Hegenbarth, F., Kim, A.C.: A geometric study of Sieradski groups. Algebra Colloq. 5(2), 203-217 (1998)

6. Cristofori, P., Mulazzani, M., Vesnin, A.: Strongly-cyclic branched coverings of knots via $(g, 1)-$ decompositions. Acta Math. Hung. 116, 163-176 (2007)

7. Doll, H.: A generalized bridge number for links in 3-manifold. Math. Ann. 294, 701-717 (1992)

8. Fox, R.H.: Construction of simply connected 3-manifolds. In: Topology of 3-Manifolds and Related Topics, Proc. The Univ. of Georgia Institute, pp. 213-216. Prentice-Hall, Englewood Cliffs (1961)

9. Gordon, C.M., Heil, W.: Simply-connected branched coverings of $S^{3}$. Proc. Am. Math. Soc. 35(1), 287288 (1972)

10. Haken, W.: Some special presentations of homotopy 3-spheres. In: Topology Conference, vol. 375, pp. 97-107. LNM (1973)

11. Hong, S., Kalliongis, J., McCullough, D., Rubinstein, J.H.: Diffeomorphisms of Elliptic 3-Manifolds. In: Lecture Notes in Mathematics, vol. 2055 (2012)

12. Johansson, I.: Über singuläre Elementarflächen und das Dehnsche Lemma. Math. Ann. 110, 312-320 (1935)

13. Lozano, Á., Vigara, R.: On the subadditivity of Montesinos complexity of closed orientable 3-manifolds. RACSAM 109(2), 267-279 (2015)

14. Lozano, Á., Vigara, R.: Representing knots by filling Dehn spheres. J. Knot Theory Ramif. 26, 1650018 (2016)

15. Lozano, Á., Vigara, R.: The triple point spectrum of closed orientable 3-manifolds. arXiv:1412.1637

16. Lozano, Á., Vigara, R.: Representing 3-Manifolds by Filling Dehn Surfaces. World Scientific, New Jersey (2016)

17. Montesinos-Amilibia, J.M.: Sobre la conjetura de Poincaré y los recubridores ramificados sobre un nudo, PhD Thesis, Universidad Complutense de Madrid (1971)

18. Montesinos-Amilibia, J.M.: Classical Tessellations and Three-Manifolds. Universitext. Springer, Berlin (1987)

19. Montesinos-Amilibia, J.M.: Representing 3-manifolds by Dehn spheres. In: Contribuciones Matemáticas: Homenaje a Joaquín Arregui Fernández, Editorial Complutense, pp. 239-247 (2000)

20. Papakyriakopoulos, C.D.: On Dehn's lemma and the asphericity of knots. Ann. Math. 2(66), 1-26 (1957)

21. Perelman, G.: Finite extinction time for the solutions to the Ricci flow on certain three-manifolds. arXiv:math/0307245

22. Seifert, H., Threlfall, W.: A Textbook of Topology. Academic Press, New York (1980)

23. Sieradski, A.J.: Combinatorial squashings, 3-manifolds, and the third homology of groups. Invent. Math. 84(1), 121-139 (1986)

24. Shima, A.: Immersions from the 2-sphere to the 3-sphere with only two triple points. Topology of real singularities and related topics (Japanese) (Kyoto, 1997). Sūrikaisekikenkyūsho Kōkyūroku 1006, 146160 (1997)

25. Vigara, R.: A new proof of a theorem of J. M. Montesinos. J. Math. Sci. Univ. Tokyo 11, 325-351 (2004)

26. Vigara, R.: A set of moves for Johansson representation of 3-manifolds. Fundam. Math. 190, 245-288 (2006)

27. Vigara, R.: Representación de 3-variedades por esferas de Dehn rellenantes, PhD Thesis, UNED (2006) 